\title{
Die Bedeutung der italienischen Kammerkantate für
}

\section{Maria Antonia Walpurgis von Bayern als Interpretin und Sammlerin von 1747 bis 1763}

Die Musikaliensammlung Maria Antonia Walpurgis' (1724-1780), Tochter des bayerischen Kurfürsten Karl Albrecht von Wittelsbach und der Erzherzogin Maria Amalia von Habsburg, ist eine der bedeutendsten Musiksammlungen in Europa, und sie besteht zum großen Teil aus Vokalmusik. Dies liegt vor allem daran, dass Maria Antonia selbst als Dichterin, Sängerin und Opernkomponistin tätig war. Im vorliegenden Aufsatz wird auf die Rolle der Gattung der italienischen Kammerkantate innerhalb der Sammlung in Bezug auf die Tätigkeit der Aristokratin als Interpretin und Sammlerin näher eingegangen.

Heute liegt die Musikaliensammlung Maria Antonias, die 1747 durch ihre Heirat mit Friedrich Christian zur sächsischen Kurprinzessin und ab 1763 zur Kurfürstin und dann Kurfürstinwitwe wurde, zu großen Teilen in der Sächsischen Landesbibliothek - Staats- und Universitätsbibliothek (SLUB) in Dresden. Im Gesamtkatalog der Sammlung, der um 1780 von dem persönlichen Sekretär Maria Antonias, Peter August, verfasst wurde, ${ }^{1}$ finden sich nicht weniger als etwa 31 Einträge zu musikalischen Quellen mit Kantaten. Großenteils stammen diese Kantaten aus der Feder von in Dresden oder in München tätigen Komponisten. Einige von ihnen, wie Johann Adolf Hasse, Giovanni Alberto Ristori, Giovanni Domenico Ferrandini und Johann Gottlieb Naumann, unterhielten sehr enge Beziehungen zur Fürstin. Ein Teil dieser Kantaten wurde nachweislich von der Maria Antonia selbst aufgeführt - dies kann anhand der musikalischen Quellen oder anderer zeitgenössischer Quellen (z.B. Hofjournale oder Briefe) nachgewiesen werden -, bei anderen Kantaten war Maria Antonia entweder die Textautorin oder die Widmungsträgerin.

Die Sammlung umfasst aber auch eine Reihe von Kantaten, die von Autoren vertont wurden, die an anderen Orten arbeiteten und mit Dresden augenscheinlich keine Verbindung hatten. In diesem Zusammenhang sind die Kantaten von Giovanni Battista Pergolesi aus Neapel und Benedetto Marcello aus Venedig besonders interessant.

Mit dem Problem der Kopistenhandschriften sowie der Datierung der Dresdner Abschriften von Hasses Kantaten hat sich bereits Ortrun Landmann in ihrem Katalog der Dresdner 
Hasse-Handschriften ausführlich beschäftigt. ${ }^{2}$ Wichtige Informationen zur Textautorschaft und zum Aufführungskontext der Hasse- und Ristori-Kantaten sowie zur chronologischen Ordnung der Kataloge von Maria Antonias Musikaliensammlung liefert Christine Fischer in ihrer Monographie über Maria Antonia als Autorin und Darstellerin. ${ }^{3}$ Über die Herkunft bzw. die Datierung der Ferrandini-Kantaten hat die Verfasserin selbst einen Beitrag für den Band der Kongressberichte La cantata da camera e lo stile galante (Treviglio 2013) geschrieben, in dem sie feststellte, dass die Handschriften sämtlich aus München stammen und später nach Dresden geschickt wurden. ${ }^{4}$

Im Folgenden werden die Kantaten von bestimmten in der Sammlung vertretenen Autoren untersucht, um einen Überblick über die verschiedenen Provenienzen der Handschriften zu geben. Was die Dresdner und Münchner Handschriften angeht, werden die bereits vorhandenen Informationen zusammengetragen und einige Hypothesen über die Rolle der Kantaten innerhalb der Sammlung formuliert. Was die anderenorts angefertigten Handschriften betrifft, hat die Verfasserin vor, ihren Verbreitungs- und Vermittlungsprozess nach Dresden sowie den Gebrauch, den die Maria Antonia von diesen Kantaten gemacht haben könnte, zu untersuchen.

Die Untersuchung fokussiert auf die Jahre von 1747 bis 1763, den Zeitraum zwischen Maria Antonias Ankunft in der sächsischen Hauptstadt und ihrer Erhebung zur Kurfürstin nach dem Tod Augusts III. Ein Teil der in der Sammlung aufbewahrten Handschriften wurde von der Wittelsbacherin vor 1747 gesammelt, als sie noch in München lebte; den restlichen Teil sammelte sie in Dresden. Die von München nach Dresden mitgebrachten Musikalien sind im Catalogo De' libri Numerati Musicali ${ }^{5}$ verzeichnet, ein Teil der anderen im Catalogo Dei Libri di Musica con i numeri negri ${ }^{6}$, den sie bis 1753 führte. ${ }^{7}$ Im oben genannten Gesamtkatalog der Sammlung sind die meisten der in diesen beiden Katalogen verzeichneten Werke sowie andere Werke und Kantaten, welche die Fürstin offensichtlich nach 1753 erwarb, aufgelistet. Dieser Katalog umfasst jedoch

2 Vgl. Ortrun Landmann, Katalog der Dresdener Hasse-Musikhandschriften. Die handschriftlich überlieferten Kompositionen von Johann Adolf Hasse (1699-1783) in der Sächsischen Landesbibliothek, Staats- und Universitätsbibliothek Dresden (CD-ROM-Ausgabe mit Begleitband), hrsg. von der RISM Arbeitsgruppe Deutschland, München 1999.

Dieselbe Verfasserin hat die zusammengestellten Ergebnisse später in einer breiteren Studie überarbeitet: Ortrun Landmann, Über das Musikerbe der sächsischen Staatskapelle. Drei Studien zur Geschichte der Dresdner Hofkapelle und Hofoper anhand ihrer Quellenüberlieferung in der SLUB Dresden, Dresden ${ }^{2} 2010$. Im Weiteren wird diese neuere Studie zitiert.

3 Vgl. Christine Fischer, Instrumentierte Visionen weiblicher Macht. Maria Antonia Walpurgis' Werke als Bühne politischer Selbstinszenierung, Kassel u. a. 2007 (Schweizer Beiträge zur Musikforschung. 7). Zu den Katalogen siehe auch den aktuellen Beitrag „Cataloghi, numeri, Schräncke und Fächer. Zur Musikaliensammlung und den historischen Noteninventaren der sächsischen Kurfürstin Maria Antonia Walpurgis'“ von Nina Eichholz im vorliegenden Band.

4 Vgl. Nastasja Gandolfo, „Le cantate da camera di Giovanni Ferrandini nella collezione della principessa Maria Antonia a Dresda“, in: La cantata da camera e lo stile galante. Sviluppi e diffusione della "nuova musica" tra il 1720 e il 1760, hrsg. von Giulia Giovani und Stefano Aresi, Amsterdam 2016, S. $27-37$.

5 D-Dl: Bibl.Arch.III.Hb,Vol.787.e, S. 1-100.

6 D-Dl: Bibl.Arch.III.Hb,Vol.787.e, S. 127-134.

7 Zu dieser Schlussfolgerung kommt Christine Fischer, weil die zwei von Maria Antonia komponierten Musikdramen Il trionfo della fedeltà (1754) und Talestri (1760) sowie andere Werke, die nach 1753 entstanden, hier nicht aufgelistet sind. Vgl. Fischer, Instrumentierte Visionen 2007 (wie Anm. 3), S. 177 f. 
auch nicht die vollständige Sammlung. ${ }^{8}$ Jóhannes Ágústsson hat vor Kurzem ein weiteres Verzeichnis im Sächsischen Hauptstaatsarchiv entdeckt, das auf den 20. Mai 1748 datiert und weitere Werke, die Maria Antonia aus München mitbrachte, enthält. Am Ende des Katalogs erscheint folgende Anmerkung des Oberhofmeisters Hugo Wilhelm Freiherr von Wetzel:

„Daß von Ihro Hoheit der König: Pohlnischen und Sächßischen Chur-Prinzeßin, gebohrene Kaÿ: Chur-Baÿerischen Prinzeßin, meiner [fol. 382b] gnädigsten frauen alles in gegen wärtigen Inventario ordentlich specifirtes aus Baÿern hieher mitgebracht worden, wirdt von Ambts wegen, durch eigene meine Hand und Unterschrifft, dann beÿgedruckten meinem Insigel bestättiget. Dreßden den 20. May, 1748. Hugo Wilhelm Freyherr von Wetzel S. ${ }^{\mathrm{r}}$ König: Hoheit der Chur Prinzeßin, ObristHoffmeister. ${ }^{\text {“9 }}$

Im Folgenden werden die Kantaten in dieser Reihenfolge untersucht:

a) Kantaten von Dresdner Autoren (Hasse, Ristori, Schürer)

b) Kantaten von Münchner Autoren (Ferrandini)

c) Kantaten von anderenorts tätigen Autoren (Pergolesi, Marcello)

Dabei soll zwischen den Kantaten, die von Maria Antonia gedichtet bzw. gesungen wurden, und den Kantaten, die ihr gewidmet wurden, unterschieden werden.

Unter den Kantaten von Dresdner Autoren, die bekanntermaßen von Maria Antonia aufgeführt wurden, erinnert man vor allem die zwei Huldigungskantaten des Hofkapellmeisters Johann Adolph Hasse (1699-1783) Grande Augusto (für Sopran, Flöten, Streicher und Basso continuo) und Che ti dirò Regina (für Sopran, Flöten, Oboen, Hörner, Streicher und Basso continuo), die jeweils zum Namenstag Augusts III. bzw. des Kurfürsten Friedrich August II. von Sachsen (3. August 1747) oder zum Geburts- und Namenstag seiner Gemahlin Maria Josepha (8. Dezember 1747) komponiert wurden. Zu diesen beiden Kantaten lieferte Maria Antonia selbst den Text. Die Kantaten sind in zwei zusammengebundenen Partiturabschriften des Hofkopisten Matthäus Schlettner erhalten (Abb. 1). ${ }^{10}$ Auf dem Einband erkennt man klar das Wappenmonogramm Maria Antonias (Abb. 2).

Auf der Schlettner-Abschrift wird das genaue Kompositionsjahr der Kantaten nicht erwähnt. Die Datierung der Kantate Che ti dirò Regina lässt sich einer anderen Abschrift, die vom Hofkopisten Johann George Kremmler angefertigt wurde und aus der Sammlung der Königin Maria Josepha stammt, entnehmen. ${ }^{11}$ Der Titel der Handschrift lautet wie folgt:

8 Es fehlen z. B. die beiden Kantaten Trova un sol mia bella Clori und Già la notte s'avvicina von Ferrandini (in D-Dl: Mus.1-J-3,2), die sicher Maria Antonia gehört haben.

9 Catalogo di Libri senza numeri, D-Dla: 10025 Geheimes Konsilium, Loc. 5220/01. Freundliche Auskunft von Jóhannes Ágústsson. Eine Transkription des Inventars bietet Appendix 5 des Beitrags von Jóhannes Ágústsson („The Saxon Crown Prince Friedrich Christian and Music. The Dresden Diaries and Account Books“) im vorliegenden Band; eine eingehendere Besprechung, die der Autorin bei der Fertigstellung ihres Beitrags noch nicht vorlag, ist im Beitrag von Nina Eichholz (wie Anm. 3) nachzulesen.

10 D-Dl: Mus.2477-J-3,1; D-Dl: Mus.2477-J-3,2. Vgl. Landmann, Über das Musikerbe 2010 (wie Anm. 2), S. 43.

11 D-Dl: Mus.2477-J-4. Vgl. ebd., S. 41. 
"Cantata Per il felicissimo Giorno di Nascita, e di Nome della $\mathrm{M}^{\mathrm{a}}$ della Regina di Polonia Elettrice di Sassonia composta da Sua Altezza Reale la Principessa Elettorale messa in musica da Giov. Adolfo Hasse 1747.“

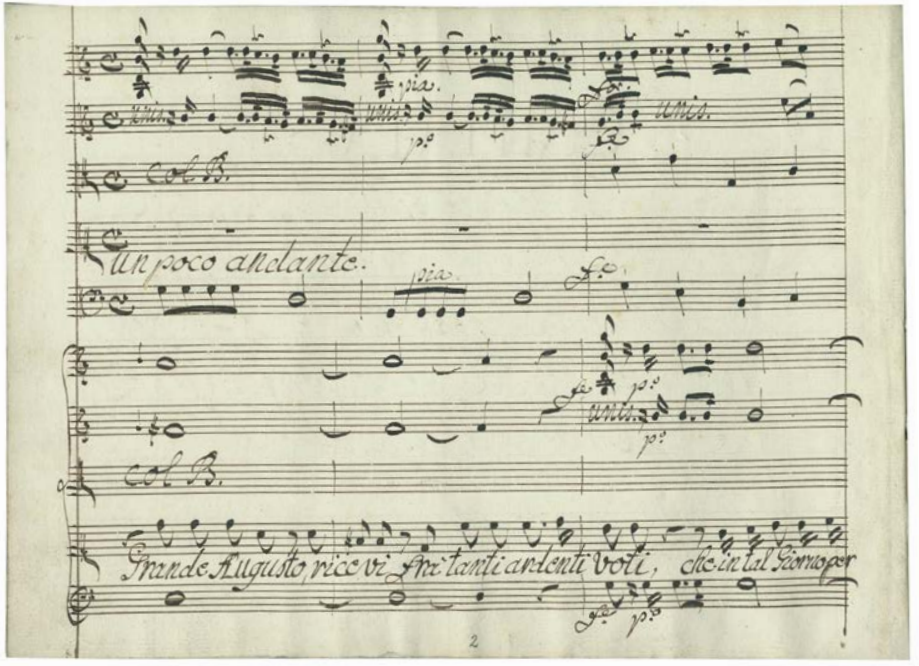

Abb. 1: J.A. Hasse, Grande Augusto ricevi, 1. Rezitativ, D-Dl: Mus.2477-J-3,1, S. 2

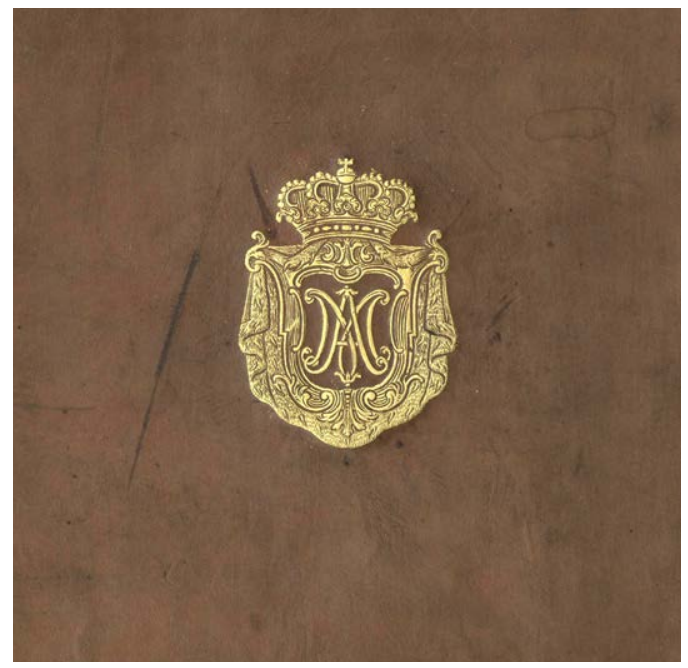

Abb. 2: Wappenmonogramm der Fürstin Maria Antonia, D-Dl: Mus.2477-J-3, Einband

Das Kompositionsjahr der Kantate Grande Augusto ricevi wird aus dem Dresdner Hofjournal ersichtlich. Im Eintrag zum 3. August 1747 wird berichtet, dass Maria Antonia die Kantate beim Festmahl in Sedlitz selbst gesungen hat:

„Vor der Tafel mit 18 Gedecken ... hat man eine Ital: Cantate angehöret, die Ihro Königl: Hoheit die Churprinzeßin selbst abgesungen, wobei einige Königl: Musici accompagniret."12

Maria Antonia übersiedelte am 20. Juni 1747 nach Dresden, infolge ihrer Hochzeit mit dem Kurprinzen Friedrich Christian von Sachsen. Da es ihr erstes Jahr am Dresdner Hof war, wollte sie mit diesen beiden Huldigungskantaten offensichtlich ihre Ehrerbietung ihren neuen Schwiegereltern gegenüber erweisen sowie ihnen eine Kostprobe ihrer Gesangskunst bieten. Es wurden Abschriften von diesen Kantaten für andere Sammlungen verfasst, die aber wahrscheinlich nicht zur Aufführung dienten. ${ }^{13}$

12 D-Dla: Oberhofmarschallamt, O I, „Dreßdener Hof-Journale“, Eintrag zum 3. August 1747.

13 Dazu zählen die zwei zusammengebundenen Abschriften aus der Königlichen Öffentlichen Bibliothek zu Dresden, die nach der Vorlage der Schlettner-Abschriften kopiert wurden (heute D-Dl: Mus.2477-J-2,1 und Mus.2477-J-2,2), oder das aus der Sammlung von Friedrich Chrysander in der Staats- und Universitätsbibliothek Carl von Ossietzky in Hamburg stammende Konvolut (D-Hs: M/A 468). Für welche Sammlungen diese Handschriften ursprünglich angefertigt wurden, lässt sich nicht genau ermitteln. Da sie den Stempel der Königlichen Privat-Musikaliensammlungen nicht aufweisen, ist zu vermuten, dass sie ursprünglich zur Nebensammlung eines nichtregierenden Mitglieds der Wettiner Dynastie gehörten und im Rahmen einer späteren Eingliederung dieser Nebensammlung in die Königlichen Privat-Musikaliensammlungen als Dubletten ausgesondert wurden. Vgl. Roland Dieter Schmidt-Hensel, „Anmerkungen zu den Schreibern und zum Quellenwert der Hassiana in der Staats- und Universitätsbibliothek Hamburg“, in: Hasse-Studien 6 (2006), S. 20-51, hier S. 23. 
Den Text der Kantate Grande Augusto präsentierte Maria Antonia außerdem im Juli 1747 als Bewerbungsstück, um in die Accademia dell'Arcadia in Rom aufgenommen zu werden. In einem Brief vom 11. Januar 1748 beglückwünschte der Prinz Sigismondo Gonzaga die Fürstin zu ihrer Dichtung mit folgenden Worten:

„Tra le cose che in quella meravigliosa Residenza mi allettarono una fra le altre vi fu l'universale applauso, che da molti letterati di quella insigne accademia dei Pastori arcadi, e da moltissimi altri, si faceva sopra la gentile ed elegante composizione italiana fatta dalla Ser.ma Reale Altezza Vostra per gli tre d'agosto passato, per il faustissimo giorno di nome di Sua Altezza Real Maestà. Intesi in oltre qualmente ella era stata acclamata a pieni voti da quegli accademici qual Pastorella arcade, sotto il nome di Ermelinda Talea“" ${ }^{14}$

Maria Antonia schrieb auch die Texte zu den drei Kantaten Didone abbandonata (1748), Lavinia a Turno (1749) und Nice a Tirsi (1749), die von dem Dresdner Kammerkomponisten Giovanni Alberto Ristori (1692-1753) in Musik gesetzt wurden. Die Kantate Didone abbandonata wurde später auch von Antonio Tozzi (ab 1774 Hofkapellmeister in München) und Johann Gottlieb Naumann (ab 1786 Oberkapellmeister in Dresden) vertont, die Lavinia a Turno von Giovanni Marco Rutini (Prag 1756) und von dem preußischen Hofkapellmeister Carl Heinrich Graun. ${ }^{15}$

Die drei Ristori-Kantaten liegen in Partiturabschriften des Hofkopisten Johann George Kremmler vor (Abb. 3). ${ }^{16}$ Das Kompositionsjahr der drei Kantaten wird auf der Titelseite der Handschriften genau angegeben. Neben den Partituren sind zu den Kantaten auch jeweils Stimmen für zwei Violinen, Viola, zwei Oboen sowie Fagott in Kremmlers Handschrift überliefert. ${ }^{17}$ Der Einsatz der Holzbläser variiert von Kantate zu Kantate. Was Didone abbandonata angeht, sind die Oboen- und Fagottstimmen in der Partitur nicht angezeigt. Aus dem Vergleich mit den Stimmensätzen geht aber hervor, dass sie in der Ouvertüre (Marcia) und in den Ritornellen der Arien spielen, indem die Oboen jeweils die erste und die zweite Violine doppeln, während der Fagott die Basslinie spielt.

In der Kantate Lavinia a Turno wird das Verhältnis zwischen den Oboen- und den Streicherstimmen etwas subtiler. In den Arien sind die Oboenstimmen in der Partitur vollständig ausgeschrieben. Hier duplieren sie jeweils die erste und die zweite Violine in den Ritornellen, so wie bei

14 Brief von Sigismondo Gonzaga an Maria Antonia (11. Januar 1748). D-Dla: 12528, Fürstennachlässe Maria Antonia, Nr. 72g. Vgl. auch Fischer, Instrumentierte Visionen 2007 (wie Anm. 3), S. $50 \mathrm{ff}$.

15 Die Lavinia-Kantate von Rutini ist die erste Fassung der Kantate, die 1756 von Johann Gottlob Immanuel Breitkopf in Leipzig gedruckt wurde. Es folgte 1762 die Veröffentlichung der Graun-Kantate. Rutini weilte 1754 in Dresden, wo er vermutlich Kenntnis des Textes erhielt, und als er ihn 1756 in Prag vertonte, lebte er unter der Obhut Maria Antonias. Mit dem Kompositionsauftrag der Graun-Kantate hatte die Fürstin hingegen wahrscheinlich nichts zu tun, da ihre Kontakte zu dem preußischen König Friedrich II. erst 1763 begannen. Es gab vermutlich auch eine Vertonung der Kantate von Hasse, die heute verschollen ist. Diese wird in der 1772 gedruckten Sammlung ausgewählter Werke Maria Antonias erwähnt: Maria Antonia Walpurgis: Vari componimenti per musica di Ermelinda Talea Pastorella Arcade Calamo ludimus, Rom u.a. 1772, S. 114.

16 D-Dl: Mus.2455-J-1 (Lavinia a Turno); Mus.2455-J-2 (Didone abbandonata); Mus.2455-J-3 (Nice a Tirsi). Vgl. Landmann, Über das Musikerbe 2010 (wie Anm. 2), S. 212 (Abb. I.2).

17 D-Dl: Mus.2455-J-1a (Lavinia a Turno); D-Dl: Mus.2455-J-2a (Didone abbandonata); D-Dl: Mus.2455-J-3a (Nice a Tirsi). 


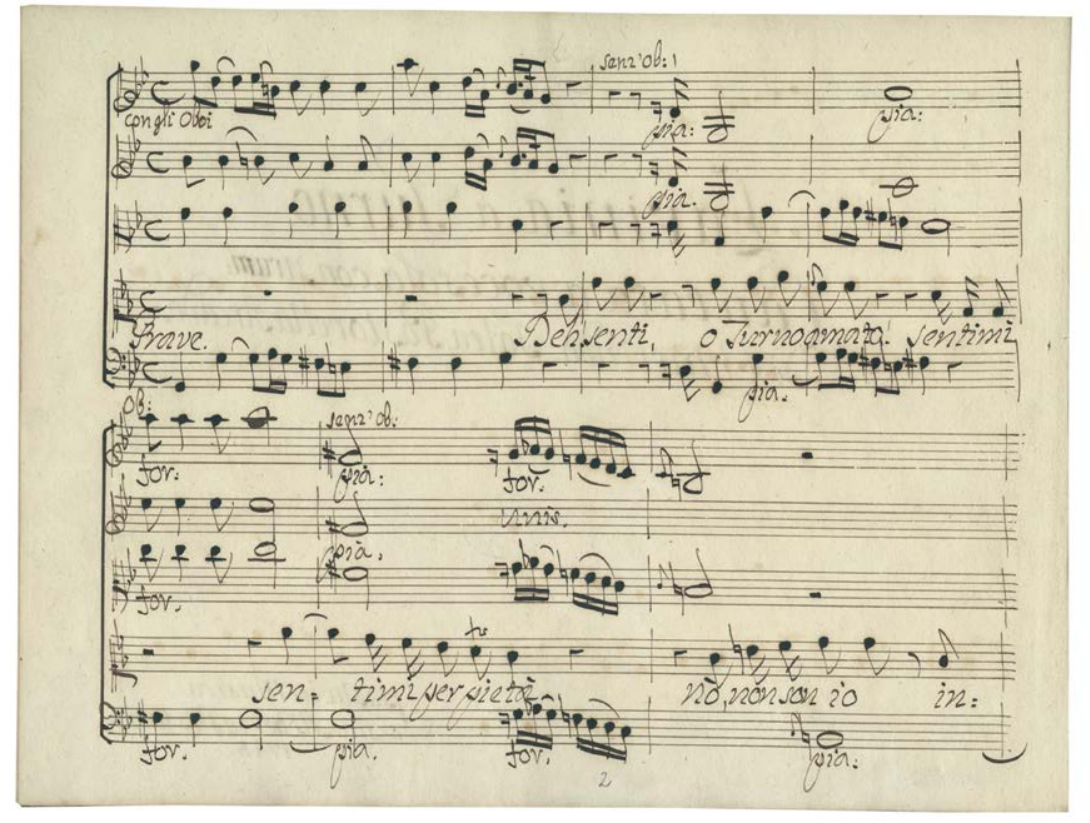

Abb. 3: G. A. Ristori, Lavinia a Turno, 1. Rezitativ, D-Dl: Mus.2455-J-1, S. 2

Didone abbandonata; daneben spielen sie auch das Ripieno. In den Rezitativen spielen sie auch teilweise zusammen mit den Violinen: Hier sind die Teile in den Stimmensätzen ausgeschrieben und in der Partitur erscheint die Anmerkung „con oboi “ an den entsprechenden Stellen oberhalb der Stimme der ersten Violine. Das Fagott doppelt die Bassstimme in den Ritornellen der Arien sowie zum Teil auch in den Rezitativen.

In der Kantate Nice a Tirsi erscheinen die Holzbläserstimmen nicht direkt in der Partitur, mit Ausnahme des „oboe concertato“ in der zweiten Arie. Ansonsten verhalten sich die Oboen ähnlich wie in Lavinia a Turno bis auf die zweite Arie: Im ersten Rezitativ spielen sie zusammen mit den Violinen in den Pausen zwischen den Sätzen des Gesangs, in der ersten Arie doppeln sie die Violinen in den Ritornellen und im zweiten Rezitativ schweigen sie. In der zweiten Arie wird eine Solopartie für die erste Oboe als „oboe concertato“ bestimmt, während die zweite Oboe die zweite Violine in den Ritornellen dupliert. Das Fagott doppelt die Bassstimme in den Rezitativen und in den Ritornellen der Arien.

Über die Uraufführungen der Kantaten Didone abbandonata und Lavinia a Turno berichtet der Oberhofmeister Graf Joseph Anton von Wackerbarth in seinem Briefwechsel mit dem sächsischen Premierminister Heinrich von Brühl. Zur Uraufführung der Didone-Kantate im privaten Musikzimmer der Fürstin im Oktober 1748 schreibt er:

„Dimanche après les Vêpres il y eut Musique dans les appartements de Madame la Princesse Electorale, où cette adorable Princesse chanta une Cantata composée par Elle-même sur un sujet, qui a été traité par différents poètes; mais il y a peu de poètes italiens, qui puissent se vanter d'y avoir aussi bien réussi que S. A. R. le."18

18 Brief vom 9. Oktober 1748. Die Briefe von Wackerbarth an Brühl aus dem Jahr 1748 sind im folgenden Band erhalten: D-Dla: 10026 Geheimes Kabinett, Loc. 3058/5, Bd. 2. 
Im Anhang des Briefes schreibt er weiter:

„Je prends la liberté de communiquer à V.E. sub rosa, c'est à dire à condition d'un secret au-dessus de tous les secrets du monde, copie de vers, que S. A. R. Mad. la Princesse E. ${ }^{\text {le }}$ a composées sur la Didone abbandonata.“

Die Kantate Lavinia a Turno wurde einen Monat später im November 1748 aufgeführt. Wackerbarth erwähnt zwei Aufführungen, jeweils am 12. und am 18. November:

„Madame la Princesse chanta hier au soir avec un gout admirable la Cantata dont j'ai l'honneur de joindre ici copie. Ristori l'a mise en musique, mais comme il m'est pas permis de nommer l'auteur de la Poésie, je laisse à Votre Excellence le plaisir d'en faire la decouverte." ${ }^{\text {"19 }}$

„Avant-hier cette adorable Princesse chanta pour la deuxième fois la cantate de Lavinia. Monseigneur le Nonce et plusieurs Dames d'entrée de la Ville firent la cour à LL. AA. RR. ${ }^{\text {les }}$ pendant cette belle Musique, qui au jugement des connoisseurs ne cède en rien à la Didone. ${ }^{\text {"20 }}$

Hasse und Ristori standen beide im nächsten Umfeld von Maria Antonia. Hasse wirkte als ihr Kompositionslehrer in ihren ersten Dresdner Jahren. Ein Lehrverhältnis zwischen Ristori und Maria Antonia ist nicht belegt, allerdings ist es gut möglich, dass die Fürstin auch bei ihm Unterricht erhielt, da er am Dresdner Hof als Lehrer wirkte. ${ }^{21}$

Die Tatsache, dass die Abschriften der Ristori-Kantaten als Unikate in der Sammlung der Fürstin überliefert sind, deutet darauf hin, dass sie außerhalb des sächsischen Hofs wenig oder gar kein Interesse erregten. Es gibt auch sonst keine Quellenbefunde, die über eventuelle Aufführungen der Kantaten außerhalb Dresdens informieren. Der Grund, weshalb Ristoris Kantaten außerhalb von Dresden anscheinend nicht zirkulierten, liegt wahrscheinlich darin, dass die Kantaten im Auftrag Maria Antonias für ihre eigene Stimme komponiert wurden. Es lässt sich leicht vorstellen, dass Maria Antonia die Handschriften der von ihr gesungenen Kantaten in ihrem Notenbestand exklusiv besitzen wollte. Ein weiterer Beleg für diese These ist, dass die Kantaten ihres Gesangslehrers Ferrandini ebenfalls als Unikate aus der Sammlung Maria Antonias bekannt sind, während die Kantate Lavinia a Turno von Carl Heinrich Graun, die auf ihren Text, aber nicht für ihre Stimme komponiert wurde, in mehreren Abschriften zirkulierte und sogar veröffentlicht wurde. Hinzu kommt, dass Ristori anderwärts als Komponist wenig bekannt war. Nach seiner Ankunft in Dresden 1717 verbrachte er fast sein ganzes Leben am sächsischen Hof und erhielt nur wenige Aufträge von anderen Höfen. ${ }^{22}$

19 Brief vom 13. November 1748.

20 Brief vom 20. November 1748.

21 Vgl. Fischer, Instrumentierte Visionen weiblicher Macht 2007 (wie Anm. 3), S. 156.

22 Es sind nur die Reisen nach Russland zu Prinzessin Anna Ivanovna (1731-1732) mit der Truppe seines Vaters und nach Neapel anlässlich der Premieren seiner Opern Temistocle (1738) und Adriano in Siria (1739) bekannt. 
Die Kantate Donna augusta perdona von Johann Georg Schürer (1720-1786) für Alt, Flöten, Hörner, Streicher und Basso continuo wurde hingegen der Fürstin gewidmet. Im Verzeichnis der Musikaliensammlung Maria Antonias erscheint sie als anonymes Werk unter der Rubrik „Musica Francese “. ${ }^{23}$ Schürer, der seine musikalische Ausbildung bereits als Jugendlicher in Dresden erhalten hatte, wirkte dort ab 1746 für die Mingotti-Operntruppe und wurde zwei Jahre später am Dresdner Hof als Kirchenkomponist angestellt. Die Kantate erklang am 15. Februar 1763, dem letzten Tag des Karnevals, an dem der Frieden von Hubertusburg abgeschlossen wurde. Sie wurde als Teil eines Divertissements aufgeführt, die zu Ehren von Maria Antonia auf Veranlassung des Grafen Charles Joseph de Marainville, der als Offizier der sächsischen Armee im Siebenjährigen Krieg (1757-1763) eingesetzt war, veranstaltet wurde. Auf dem Titelblatt des Drucks, in dem der Ablauf des Divertissements beschrieben wird, erscheint folgender Titel:

\section{„DÉTAIL D'UN DIVERTISSEMENT, DONNÉ LE DERNIER JOUR DE CARNAVAL 1763. À S. A. R. MADAME LA PRINCESSE ELECTORALE DE SAXE, PAR Mr. LE COMTE DE MARAINVILLE, Brigadier des Armées de S. M. Très-Chretiênne, Envoié à l'Armée Imperiale en Saxe. DRESDE, chez GEORG CONRAD WALTHER, Libraire du Roi. 1763.“ ${ }^{24}$}

Die Kantate liegt in zwei Partiturabschriften von Johann Gottlieb Haußstädler vor (Abb. 4). ${ }^{25}$ Die eine ist als Anhang dem oben genannten Druck beigeheftet; die andere befindet sich in einem handschriftlichen Konvolut, ${ }^{26}$ in das auch zwei französische Sololieder und ein mehrstimmiger Chor (Chœur chanté par tous les acteurs) eingebunden sind. Diese erklangen im Rahmen einer Farce pour six personnages, die im zweiten Teil des Divertissements gespielt wurde. Die ersten 32 Seiten dieses Konvoluts enthalten eine handschriftliche Kopie des Librettos.

Die italienische Kantate von Schürer wurde im Verlauf der Festlichkeiten als Gesang von Schäfern und Landsleuten zu Ehren der Fürstin aufgeführt. Sie wurde durch einen Marsch eingeleitet, der von den Instrumentalisten der Hofkapelle gespielt wurde. Diese marschierten in Zweierreihen als Schäfer und Landsleute gekleidet. Der Sänger war ein Altist aus der Hofkapelle, dessen Name leider nicht erwähnt wird. Es wird lediglich gesagt, dass er als Kind verkleidet war und dass sein Gesang den Anwesenden sehr gefiel:

„Derrière cet enfant postiche était une personne de talent de la Chapelle de Sa Majesté le Roy de Pologne, dont le visage était couvert d'une masque ressemblant à celui d'un Enfant. Il avait la tête adaptée au corps de l'Enfant et on avait arrangé un rideau pour le baldaquin pour le cacher. Il a chanté avec un art et un gout, qui a fait infiniment plaisir à la famille royale ainsi qu'à tout l'assemblée.“' ${ }^{\prime 7}$

23 D-Dl: Bibl.Arch.III.Hb,Vol.787.g, S. 134.

24 D-Dl: MT.1620,2.Rara.

25 Vgl. Landmann, Über das Musikerbe 2010 (wie Anm. 2), S. 227 (Abb. I.24).

26 D-Dl: Mus.3096-J-1.

27 D-Dl: Mus.3096-J-1, S. 7 f. 


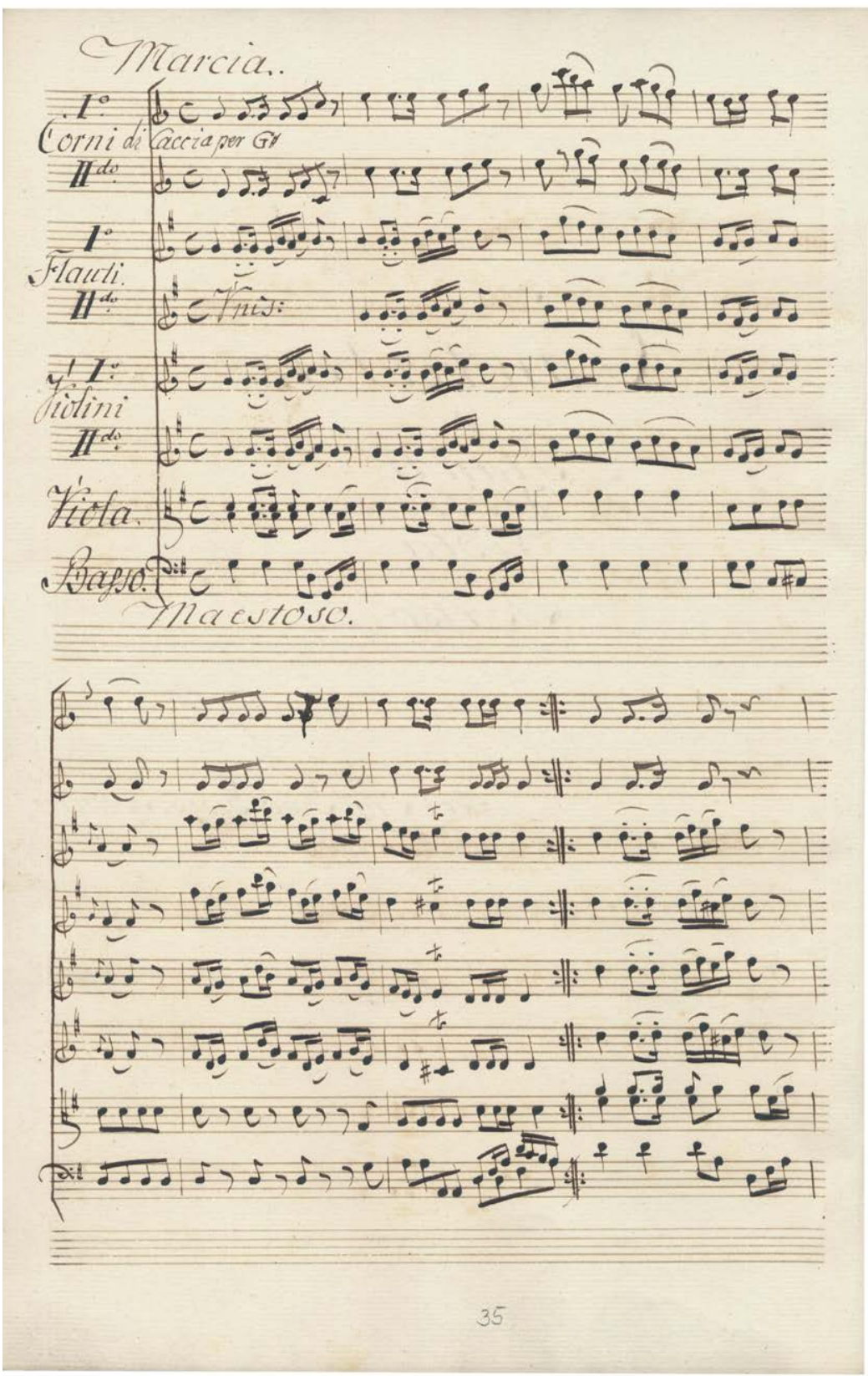

Abb. 4: J. G. Schürer, Donna augusta perdona, Ouvertüre, D-Dl: Mus.3096-J-1, S. 35

Offensichtlich war er noch ziemlich jung, weil die Dame, die die Rolle seiner Gouvernante spielte, ihm sagte:

„La Gouvernante fit à son nourrisson l'exhortation suivante. Cher Illustre Enfant, vôtre âge est si tendre, qu'on n'oseroit vous exposer à produire vos talens devant une Princesse, qui en a de supérieur en tous genres, si sa bonté et son indulgence n'egaloient son merite. [...] C'est la raison qui m’a fait vous contrarier dans les premiers désirs que vous avez eu de chanter les louanges de l'aimable Princesse, pour laquelle ce divertissement a été arrangé. “28 
Was die Kantaten von Dresdner Autoren angeht, handelt es sich meistens um Kantaten, bei denen Maria Antonia entweder die Textautorin bzw. die Sängerin oder die Widmungsträgerin war. ${ }^{29}$ Daher ist es verständlich, dass sie deren Handschriften in ihrer Bibliothek zu repräsentativen Zwecken aufbewahren wollte.

Die Sammlung der Fürstin enthält auch eine beträchtliche Anzahl an Kantaten ihres ehemaligen Gesangsmeisters aus der Münchner Zeit Giovanni Domenico Ferrandini (1709-1791). Es sind aus ihrem Bestand fünf Handschriften überliefert, die insgesamt 29 Kantaten enthalten. Folgende Argumente lassen den Schluss zu, dass die Handschriften in München angefertigt wurden:

a) Die Schreiberhandschrift von vier der fünf Kantatenhandschriften ${ }^{30}$ ähnelt sehr stark derjenigen eines in der Sammlung der Kurfürstin Maria Anna in der Bayerischen Staatsbibliothek in München vertretenen Kopisten. Der Schreiber dieser vier genannten Handschriften kann fast sicher mit dem Münchner Schreiber, den Gertraut Haberkamp und Robert Münster mit der Sigle „Schreiber A“ bezeichnen, ${ }^{31}$ identifiziert werden (Abb. 5).

b) Die Handschrift der Kantate Deh chi alla tetra idea ${ }^{32}$ stammt von der Hand eines anderen Kopisten, trotzdem wurde sie sicher in München kopiert, weil das Wasserzeichen ein bayerisches Wappen darstellt (Abb. 6). Es handelt sich um ein gekröntes geviertes Wappen mit Herzschild, der von zwei Löwen gehalten wird. Obwohl es nicht gelungen ist, den genauen Papiermacher zu identifizieren, sind ähnliche Wasserzeichentypen bei den Papiermühlen von Johann Leonard Bullinger in Wiesensteig und Johann Christoph Mayr in Thalkirchen nachweisbar (Abb. 7). ${ }^{33}$

c) Die Handschrift D-Dl: Mus.3037-J-5 besteht aus drei Huldigungskantaten, die zum Namenstag des bayerischen Kurfürsten Maximilian Joseph bzw. seiner Gattin Maria Anna (des Bruders und der Schwägerin Maria Antonias) komponiert wurden.

d) In der Abschrift der Solokantate Dell'idol mio trafitto ${ }^{34}$ schrieb Ferrandini den poetischen Text von eigener Hand (Abb. 8). Dies ergibt sich aus dem Vergleich der Abschrift mit einer Ariensammlung, die Paolo Cattelan als Autograf von Ferrandini identifizierte. ${ }^{35}$ Dies bedeutet, dass die Abschrift in München vor ca. 1755, als Ferrandini München mutmaßlich verließ, kopiert wurde (Abb. 9).

29 Die meisten Kantaten, die Maria Antonia dichtete, wurden auch von ihr selbst gesungen. Es gibt aber einige Kantaten, deren Vertonung bzw. Aufführung nicht dokumentiert ist. Vgl. Fischer, Instrumentierte Visionen 2007 (wie Anm. 3), S. 442.

30 D-Dl: Mus.3037-J-1; Mus.3037-J-4; Mus.3037-J-5; Mus.3037-K-1.

31 Vgl. Gertraut Haberkamp und Robert Münster, Die ehemaligen Musikhandschriftensammlungen der Königlichen Hofkapelle und der Kurfürstin Maria Anna in München: thematischer Katalog, München 1982, S. XXV (Kataloge Bayerischer Musiksammlungen. 9).

32 D-Dl: Mus.3037-J-2.

33 Freundliche Auskunft von Frau Andrea Lothe, Deutsches Buch- und Schriftmuseum der Deutschen Nationalbibliothek Leipzig, Papierhistorische Sammlungen.

34 D-Dl: Mus.3037-J-1.

35 D-Dl: Mus.3037-J-9. Freundliche Auskunft von Prof. Paolo Cattelan, Università Ca' Foscari di Venezia. 

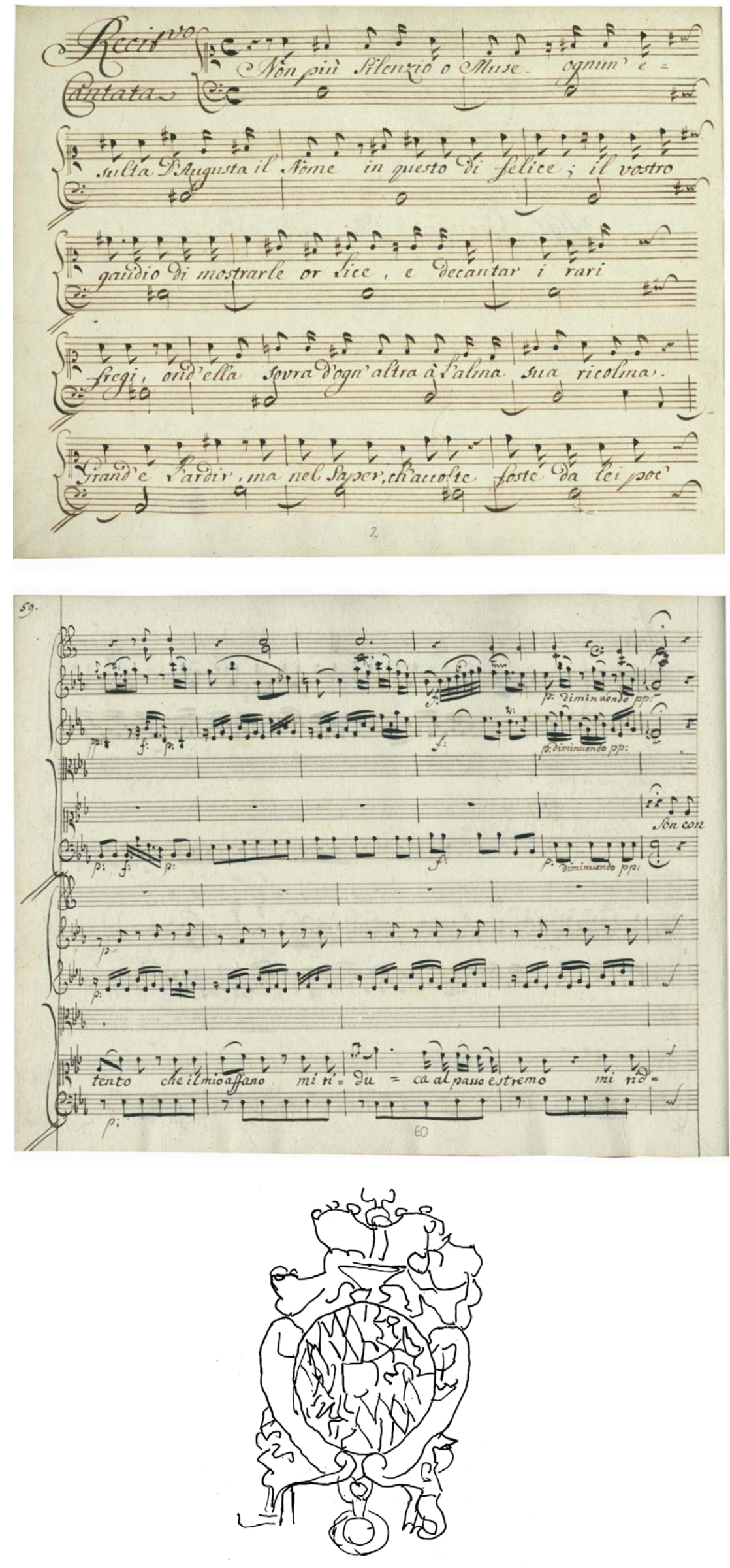

Abb. 5: G.D. Ferrandini, Non più silenzio o Muse, 1. Rezitativ, D-Dl: Mus.3037-J-5, S. 2

Abb. 6: G. D. Ferrandini, Deh chi alla tetra idea, 1 . Arie, D-Dl: Mus.3037-J-2, S. 6
Abb. 7: Wasserzeichen ,Bayerisches Wappen aus D-Dl: Mus.3037-J-2 


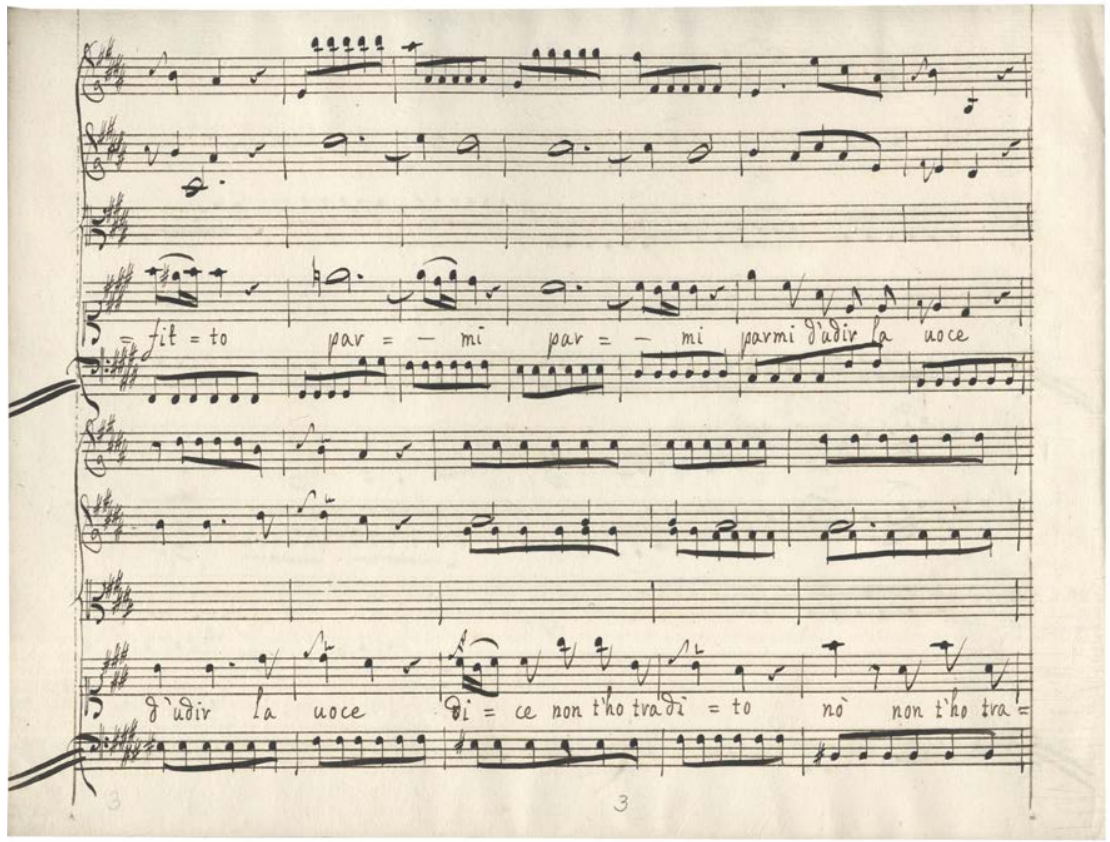

Abb. 8: G.D. Ferrandini, Dell'idol mio trafitto, 1 . Arie, D-Dl: Mus.3037-J-1, S. 3

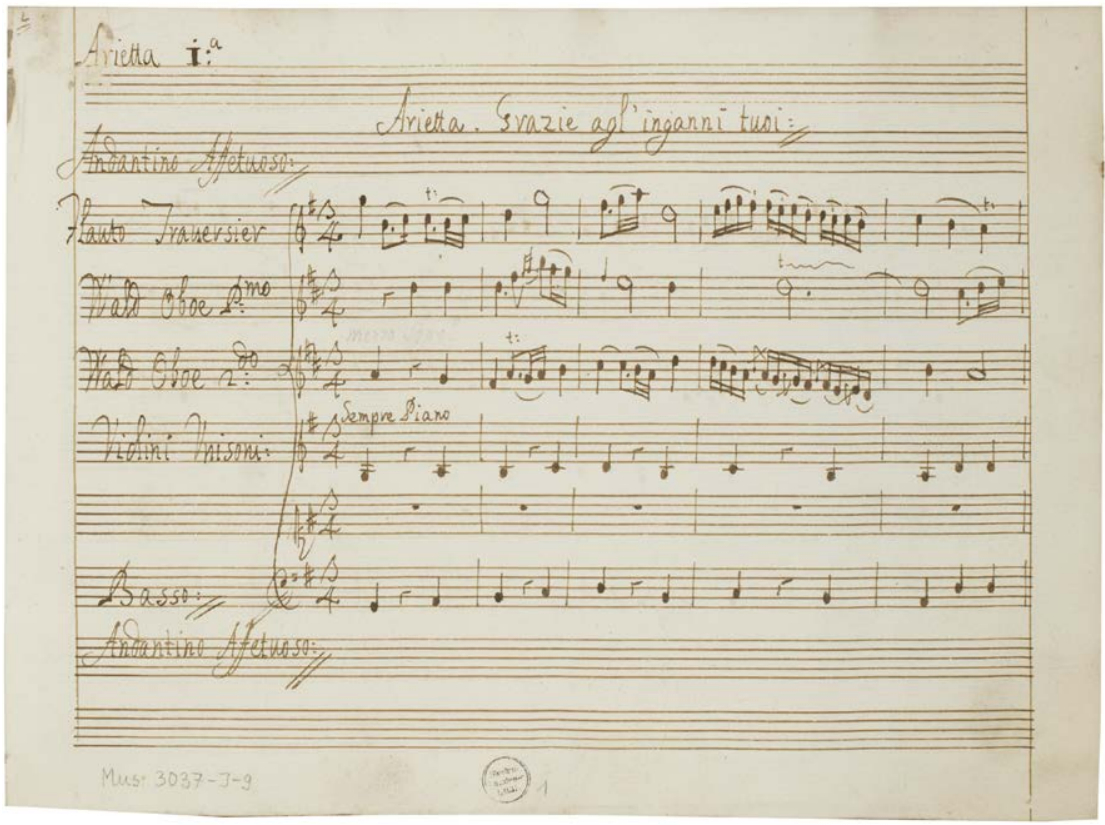

Abb. 9: G.D. Ferrandini, Grazie agli inganni tuoi alfin respiro, D-Dl: Mus.3037-J-9, S. 4

Die Ferrandini-Kantaten erscheinen aber nicht im Catalogo De'libri Numerati Musicali, der die aus München mitgebrachten Musikalien enthält, sondern erst im Catalogo Dei Libri di Musica con i numeri negri, den Maria Antonia nach ihrer Übersiedlung nach Dresden erstellte. Hier sind vier Kantatenbände von Ferrandini aufgelistet. ${ }^{36}$ Daraus geht hervor, dass die Handschriften erst nach 1747 in die Sammlung der Fürstin gelangten. Am Ende des Catalogo Dei Libri di Musica con i numeri negri ist auch eine andere Kantate von Ferrandini verzeichnet, die aber mit Bleistift von einer späteren Hand hinzugefügt wurde. ${ }^{37}$ Da Maria Antonia den Katalog ihrer eigenen Sammlung nur bis 1753 führte, ist zu vermuten, dass sie diese Kantate zu einem späteren Zeitpunkt

36 D-Dl: Bibl.Arch.III.Hb,Vol.787.e, S. 128 f.

37 Ebd., S. 134. 
erwarb. Obwohl es keinen sicheren Beweis gibt, handelt es sich wahrscheinlich um die einzelne Abschrift der Solokantate Deh chi alla tetra idea, weil diese die Handschrift eines anderen Kopisten als die übrigen Manuskripte aufweist.

Im Gesamtkatalog der Sammlung (Catalogo della Musica, e de' Libretti di S. A. R. Maria Antonia) sind schließlich alle fünf Kantatenhandschriften von Ferrandini aufgelistet. ${ }^{38}$ Da die Handschriften in eleganter Reinschrift niedergeschrieben und in prächtigen, goldverzierten Ledereinbänden gebunden sind, ist anzunehmen, dass es Kantaten sind, die Maria Antonia in ihrer Jugendzeit in München gesungen hatte, von denen sie später Abschriften als Erinnerungsstücke für ihre Sammlung verlangte. Den Einträgen im Catalogo Dei Libri di Musica con i numeri negri gemäß besaß die Fürstin ursprünglich auch die Instrumentalstimmen („stromenti cavati“) von mindestens zwei Kantaten. ${ }^{39}$ Dies könnte bedeuten, dass sie vorhatte, diese in Dresden wiederaufzuführen. Nach einem Brief des Grafen von Wackerbarth an Brühl vom 6. November 1748 sang Maria Antonia tatsächlich eine Kantate von Ferrandini in Dresden anlässlich der Feierlichkeiten für St. Hubertus am 3. November. Der Text war von ihrem Vater, Kaiser Karl VII., gedichtet worden und die Arien wurden bei der Gelegenheit als Jagdfanfaren arrangiert, da St. Hubertus als Schutzpatron der Jäger verehrt wird:

„Dimanche passé LL. AA. RR. les ont celebrée la St. Hubert d'une manière toute nouvelle, sans bouger de leurs appartements. Madame la Princesse nous donna une très belle Cantata, dont les vers étoient de la composition de S. M. l'Empereur Charles VII; la musique du $S^{\mathrm{r}}$ Ferrandini et les airs adaptés aux différents fanfares de la chasse par force. ${ }^{{ }^{4} 0}$

Um welche Kantate es sich handelt, lässt sich leider nicht mehr ermitteln. Die typischen Jagdinstrumente wie Hörner und Oboen erscheinen in der ersten Arie der Namenstagskantate für Prinz Maximilian Joseph La messaggera Dea. In der Einleitung zur Arie spielen die Hörner charakteristische Motive der Jagdfanfare wie punktierte Rhythmen und Staccato-Triolen, jedoch scheint es merkwürdig, dass eine zu einer bestimmten Gelegenheit komponierte Huldigungskantate in einem völlig verschiedenen Kontext wiederaufgeführt wird. Jagdhörner gehören auch zur instrumentalen Besetzung der ersten Arie der Namenstagskantate zu Ehren der Kurfürstin Maria Anna Non più silenzio o Muse, die aber erst nach dem Tod des Kaisers komponiert wurde. Hörner neben Flöten und Oboen finden sich weiterhin in der Kantate Deh chi alla tetra idea, wobei die Hörner nur eine Ripieno-Partie spielen. Außerdem wäre diese Kantate aufgrund der durch den Text hervorgerufenen düsteren Atmosphäre zur festlichen Gelegenheit nicht geeignet gewesen. Trotzdem ist dieser Brief ein wichtiges Zeugnis, weil er belegt, dass wenigstens eine Kantate, die Ferrandini in München komponierte, in Dresden gespielt wurde.

Schließlich umfasst die Sammlung der Fürstin verschiedene Kantatenhandschriften von italienischen Autoren, die, soweit bekannt ist, persönlich mit dem Dresdner Hof nichts zu tun hatten. So kommt man nicht umhin, sich die Frage zu stellen, wie diese Handschriften in den Besitz von Maria Antonia gelangten. Zu dieser Reihe gehören vier Kantaten des neapolitanischen Kompo-

38 D-Dl: Bibl.Arch.III.Hb,Vol.787.g, S. 123.

39 D-Dl: Bibl.Arch.III.Hb,Vol.787.e, S. 128.

40 Brief vom 6. November 1748. D-Dla: Geheimes Kabinett, Loc. 3058/5, Bd. 2. 
nisten Giovanni Battista Pergolesi (1710-1736) und die heroische Kantate Cassandra des venezianischen Komponisten Benedetto Marcello (1686-1739). Die vier Kantaten von Pergolesi (Dalsigre ah mia Dalsigre, Luce degli occhi miei, Chi non ode e chi non vede, Nel chiuso centro) sind in einer Handschrift zusammengebunden ${ }^{41}$ deren Titelseite diejenige eines Drucks der Kantaten wiedergibt, der in Neapel von dem Kontrabassisten der königlichen Hofkapelle Gioacchino Bruno veröffentlicht worden war:

"Quattro Cantate da Camere [sic!]. La prima per Cimbalo, e Tre con varii Recitativi, Violini, e Violetta obbligato. Giovan Battista Pergolese. Raccolte da Gioacchino Bruno, Contrabasso della Real Capella di Napoli. Per divertimento à Dilettanti di Musica. Opera Seconda in Rame."

Der Druck der vier Kantaten erschien erstmals 1738. Das Datum ergibt sich aus der Widmungsrede von Gioacchino Bruno an den englischen Botschafter im Königtum von Neapel-Sizilien, James Francis Fitzjames Stuart. Der auf dem Titelblatt der Dresdner Handschrift wiedergegebene Titel bezieht sich auf ein später erschienenes Druckexemplar, das von den Brüdern Giovanni und Giuseppe Palmiero in Fontana Medina zum Verkauf angeboten wurde. ${ }^{42}$

Es stellt sich die Frage, wie die Handschrift in den Notenbestand Maria Antonias gelangte. Die Achse Dresden-Neapel, die sich infolge der Heirat der sächsischen Prinzessin Maria Amalia mit König Karl IV. von Bourbon (1738) ergeben hat, kommt hier nicht infrage, weil die vier Kantaten von Pergolesi im Catalogo di Libri senza numeri verzeichnet sind..$^{43}$ Dies bedeutet, dass Maria Antonia den Band bereits in München vor 1747 besaß.

Das Vorliegen der Handschrift im Catalogo di Libri senza numeri widerlegt eine von Jóhannes Ágústsson in seinem Referat aufgestellte Hypothese; er vermutete, dass die Handschrift durch den Kurprinzen Friedrich Christian während dessen Kavalierreise in Venedig um 1740 erworben wurde. Dabei stützte er sich auf eine ihm persönlich übermittelte Auskunft von Claudio Bacciagaluppi, laut dem die verwendete Papierart auf Venedig hinweist (Abb. 10). ${ }^{44}$

Es ist daher anzunehmen, dass Maria Antonia die Handschrift direkt aus Italien erwarb. Der Einband muss allerdings später ersetzt worden sein, da die Handschrift im Catalogo di Libri senza numeri als ein „von Gold Pappier eingebundenes Buch“ beschrieben wird, während sie heute mit einem braunen Ledereinband versehen ist.

Im Gesamtkatalog der Sammlung der Fürstin ist außerdem eine Kopie der Kantate La Cassandra von Benedetto Marcello verzeichnet. ${ }^{45}$ Es bleibt die Frage, auf welche der heute vorhandenen Abschriften der Kantate sich dieser Eintrag bezieht. In der SLUB sind zwei Abschriften auf-

41 D-Dl: Mus.3005-J-1.

42 Vgl. Giulia Giovani, „Le cantate da camera edite a Napoli tra Sei e Settecento: il caso di Cataldo Amodei e Giovanni Battista Pergolesi”, in: Nicola Porpora musicista europeo. Le corti, i teatri, i cantanti, i librettisti, hrsg. von Niccolò Maccavino, Reggio Calabria 2011, S. 281-301, hier S. $294 \mathrm{ff}$.

43 Cantate del Sig. Pergolese, ein von Gold Pappier eingebundenes Buch. D-Dla: 10025 Geheimes Konsilium, Loc. 5220/01, fol. 382a.

44 In der Druckfassung seines Referats für den vorliegenden Band (wie Anm. 9) hat Jóhannes Águstsson seine frühere Hypothese revidiert.

45 D-Dl: Bibl.Arch.III.Hb,Vol.787.g, S. 126. 


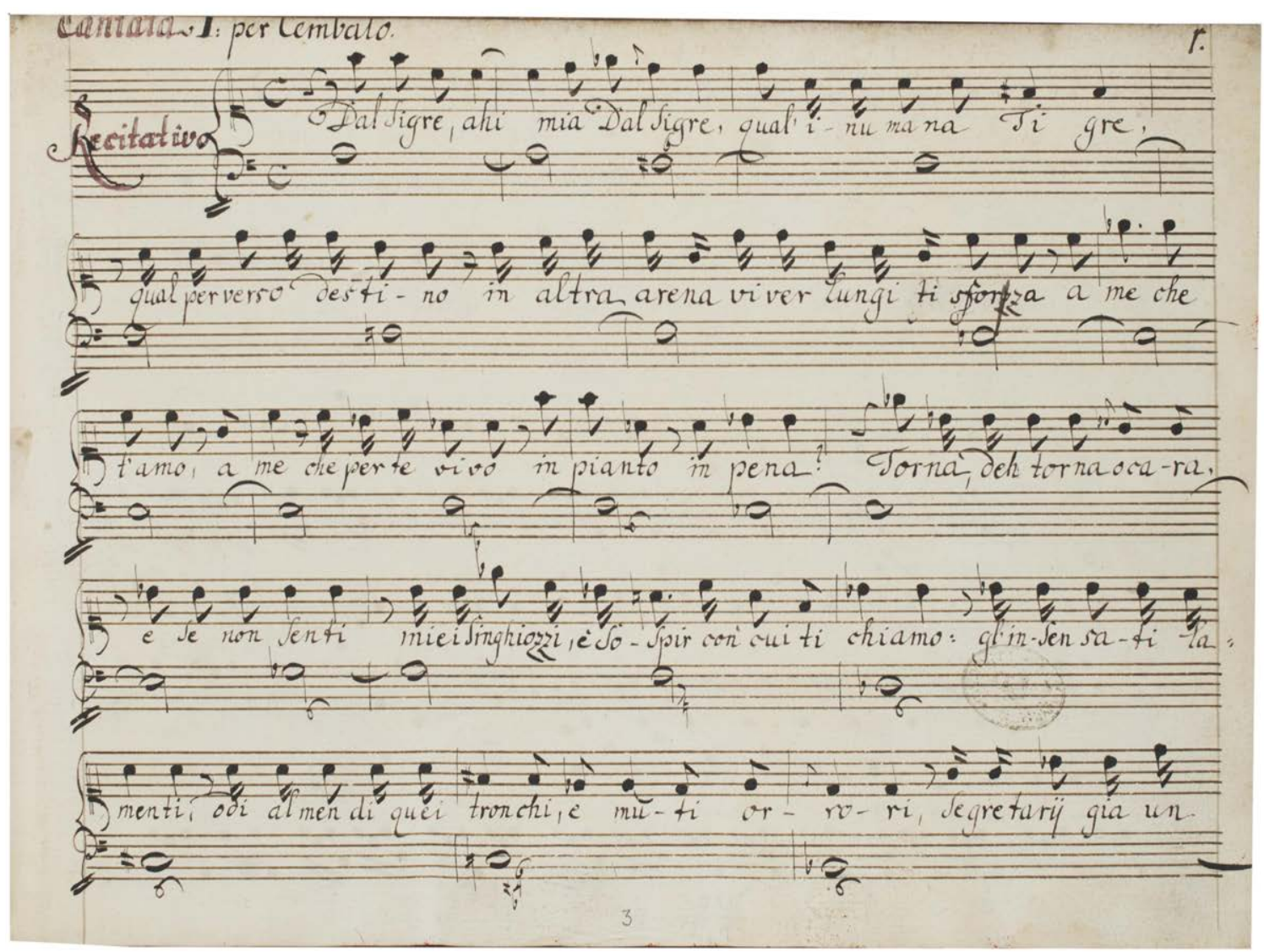

Abb. 10: G. B. Pergolesi, Quattro cantate, Titelseite, D-Dl: Mus.3005-J-1

bewahrt: Die eine ist eine Einzelabschrift vermutlich venezianischer Herkunft, ${ }^{46}$ die andere ist mit der Kantate Il Timoteo desselben Komponisten zusammengebunden und gehörte ursprünglich dem Kammerkomponisten und Kirchenorganisten des Dresdner Hofs Giovanni Alberto Ristori. ${ }^{47}$ Was die einzelne Abschrift angeht, kann man nur als Hypothese annehmen, dass sie in Venedig angefertigt wurde, weil das Papier das für die Lagunen-Region typische ,Tre lune-Wasserzeichen aufweist; sonst lässt sich nichts Genaueres über den Überlieferungsprozess sagen. Nach Jóhannes Ágústssons Forschungen lässt sich die mit Il Timoteo zusammengebundene Handschrift hingegen aufgrund des Einbands dem Besitz von Giovanni Alberto Ristori zuordnen. ${ }^{48}$ Aufgrund der persönlichen Kontakte zwischen Ristori und Maria Antonia würde es plausibel erscheinen, dass Ristori die Handschrift der Fürstin überlassen haben könnte. Eine merkwürdige Tatsache ist aber, dass Il Timoteo nirgendwo in den Katalogen der Sammlung der Fürstin erscheint. Allerdings ist, wie bereits erwähnt, keiner von diesen vollständig.

Die Entstehung beider Handschriften ist vermutlich auf Venedig zurückzuführen, wo Benedetto Marcello die Kantate 1727 auf einen Text des Abbés Antonio Conti komponierte. ${ }^{49}$ Es han-

46 D-Dl: Mus.2416-K-2.

47 D-Dl: Mus.2416-C-1a.

48 Freundliche Auskunft von Jóhannes Ágústsson.

49 Vgl. Eleanor Selfridge-Field, The music of Benedetto and Alessandro Marcello: A thematic catalogue with commentary on the composers, repertory and sources, Oxford 1990, Nr. A240b. 
delt sich um eine in formaler Hinsicht groß angelegte dramatische Kantate, die nicht eindeutig in Rezitative und Arien aufgeteilt, sondern in eine Reihe verschiedenartiger formaler Abschnitte gegliedert ist.

Es kann also behauptet werden, dass, obwohl Pergolesi und Marcello persönlich keine Beziehung zu Dresden hatten, der Wettiner Hof enge Verbindungen zu Venedig und Neapel als Zentren der Kunst und der Musik unterhielt. Weiterhin ist zu vermuten, dass Maria Antonia die Handschriften der Pergolesi- und Marcello-Kantaten im Wesentlichen aus Gründen der Erhaltung sowie der historischen Dokumentation erwarb, um ein repräsentatives Beispiel der kompositorischen Kunst der beiden Komponisten in ihrer Sammlung zu haben. Dafür spricht, dass die Kantaten in Partitur kopiert und mit Ledereinbänden versehen sind. Zur Aufführung wurden hingegen einzelne Stimmenabschriften verwendet, die aber selten aufbewahrt wurden, weil sie als nicht wertvolles Material angesehen wurden.

Abschließend kann festgehalten werden, dass Maria Antonias Sammeltätigkeit im Hinblick auf die Gattung der Kantate zwei verschiedene Richtungen verfolgte. Auf der einen Seite war diese eng verbunden mit ihrer Gesangstätigkeit, indem die Fürstin handschriftliche Partiturkopien von Kantaten, die sie entweder in München oder in ihren frühen Dresdner Jahren gesungen hatte, als Erinnerungsstücke sammelte; auf der anderen Seite sammelte Maria Antonia aus repräsentativen Gründen Abschriften von Kantaten, die ihr gewidmet wurden (z. B. Schürers Donna augusta perdona oder Naumanns La felicità dei popoli), sowie Einzel- bzw. Sammelhandschriften von Kantaten zeitgenössischer Autoren aus reinem historischen Interesse.

Im Catalogo Dei Libri di Musica con i numeri negri ist zudem dokumentiert, dass Maria Antonia auch die Instrumentalstimmen der von ihr nachweislich gesungenen Hasse- und Ristori-Kantaten sowie von mindestens zwei der Ferrandini-Kantaten besaß. Bei den Hasse- und Ristori-Kantaten stellt sich die Frage, ob sie die Stimmen als Erinnerung an die vergangenen Aufführungen aufbewahrte oder ob sie die Absicht hatte, die Kantaten erneut aufzuführen. Im Fall der FerrandiniKantaten ist es ziemlich naheliegend, dass sie im Sinn hatte, diese auch in Dresden aufzuführen.

Wenn man die vokalen und instrumentalen Eigenarten der verschiedenen von der Prinzessin gesungenen Kantaten analysiert, bemerkt man eine allmähliche Entwicklung der technischen Schwierigkeit sowie eine progressive Erweiterung der instrumentalen Besetzung, von Ferrandinis Kantaten für Solo-Sopranstimme und Basso continuo über die Kantaten desselben für Sopran und Streicher, bis zur beinahe orchestralen Besetzung der Kantaten von Hasse und Ristori. Auf der Grundlage dieser Überlegungen kann vermutet werden, dass Ferrandini die Kantaten für Sopran und Basso continuo (in: D-Dl: Mus.3037-K-1) für Maria Antonia in ihren frühen Studienjahren um 1740 komponierte. Die in diesem Band enthaltenen Kantaten sind aus einer anderen Kantatensammlung von Ferrandini, die 1739 entstand, herausgezogen und gegebenenfalls für Sopranstimme transponiert worden. ${ }^{50}$ In diesen Kantaten bewegt sich der Stimmambitus innerhalb der Oktave und der Gesamtumfang geht vom eingestrichenen $c$ zum zweigestrichenen $g$. Die übrigen Ferrandini-Kantaten sowie die Hasse- und Ristori-Kantaten weisen hingegen nicht nur einen größeren Stimmumfang auf, sondern auch breite und komplexe Verzierungen in den Arien sowie schwierige Sprünge (z.B. eines Tritonus oder einer Septe) in den Rezitativen. Daraus geht hervor,

50 Die Sammlung wurde Kaiser Karl VI. gewidmet und liegt heute in der Österreichischen Nationalbibliothek in Wien vor (A-Wn: Mus. Hs. 19028). Siehe Gandolfo, Le cantate da camera 2016 (wie Anm. 4), S. $32 \mathrm{f}$. 
dass Ferrandini die anderen Kantaten für Maria Antonia wahrscheinlich nicht lange vor deren Übersiedlung nach Dresden komponierte. Die zunehmende technische Komplexität der Kantaten entspricht der Entwicklung des sängerischen Werdegangs der Fürstin, die um 1747 nicht mehr nur eine Amateursängerin war, sondern nach einer Professionalität strebte, die sie etliche Jahre später zeigte, als sie die jeweilige Rolle der Protagonistin in den von ihr selbst komponierten Opern Il Trionfo della fedeltà (1754) und Talestri (1760) interpretierte. 
